\title{
Erratum
}

Cytogenet Genome Res 108:364-365 (2005)

Erratum to Cytogenet Genome Res 103:235-244 (2003)

\section{Knockout mice provide novel insights into meiotic chromosome and telomere dynamics}

\author{
H. Scherthan \\ Max-Planck-Institut für Molekulare Genetik, Berlin (Germany)
}

The details ' $\mathrm{B}$ ' in Figure 1 were erroneously reproduced too dark and some spots in Fig. 2 occurred misplaced. The correct versions of Fig. $1 \mathrm{~B}$ and Fig. 2 are printed below.

Fig. 1. (A) Spread spermatocytes of wild-type and $S y c p 3^{-/-}$mice painted for mouse chromosomes (MMU) 13 (FITC; green) and stained for SCP1 transverse filament protein of the SC (Cy3, red) each show a single painted MMU13 bivalent. The MMU13 bivalent is more extended in the Sycp $3^{-/-}$spermatocyte, indicating that SYCP3 is involved in chromosome condensation (for details see Liebe et al., 2003). (B) Left detail: EM of telomere attachment plate of a $S y c p 3^{-/-}$spermatocyte showing an electron-dense plate at the nucleoplasmic face of the nuclear membrane. Fibrillar material spanning the NE emanates into the cytoplasm (black arrow) opposite of a telomere attachment (white arrowheads). Right detail: Telomere attachment after EM-ISH with $\mathrm{T}_{2} \mathrm{AG}_{3}$ repeat probe. Telomere-repeat signals (black gold grains; arrow) are located at the attachment plate lining the inner NM (upper arrowheads), indicating that telomere repeats are contained in the attachment plate. The outline of the nuclear envelope is marked by facing upper and lower arrowheads. Upper arrowheads indicate the inner nuclear membrane (the nucleus is always situated at the top of the image). Bars: $0.2 \mu \mathrm{m}$. (C) Mouse bouquet spermatocyte showing the clustering of a number of telomeres (red) near the centrosome as stained by $\gamma$-tubulin (green; arrow) at the cytoplasmic side of the nuclear top (B, C reproduced from Liebe et al., 2003). (D) Three-dimensionally preserved leptotene spermatocytes of wild-type (WT) and $\mathrm{H}_{2} \mathrm{ax}^{-/}$mice showing short SYCP3 fragments (green) and TRF1 telomere dots (red). While telomere clustering is not evident in the wild type (focal plane near nuclear top), telomeres have formed a tight bouquet in the $\mathrm{H}_{2} \mathrm{ax}^{-/-}$leptotene spermatocyte (focal plane near nuclear equator) indicating that histone $\mathrm{H} 2 \mathrm{AX}$ plays a role in regulating meiotic telomere dynamics (Fernandez-Capetillo et al., 2003a).

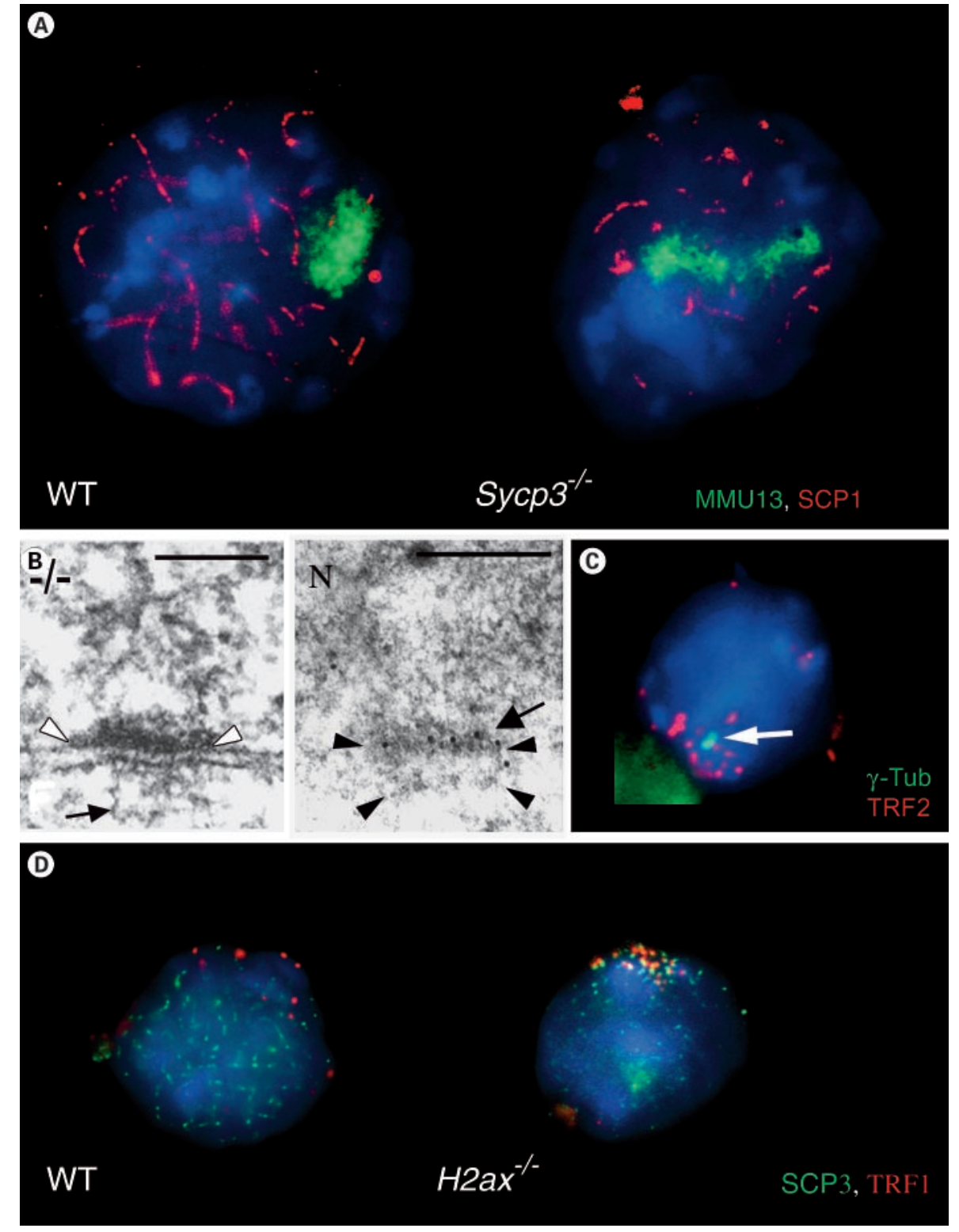




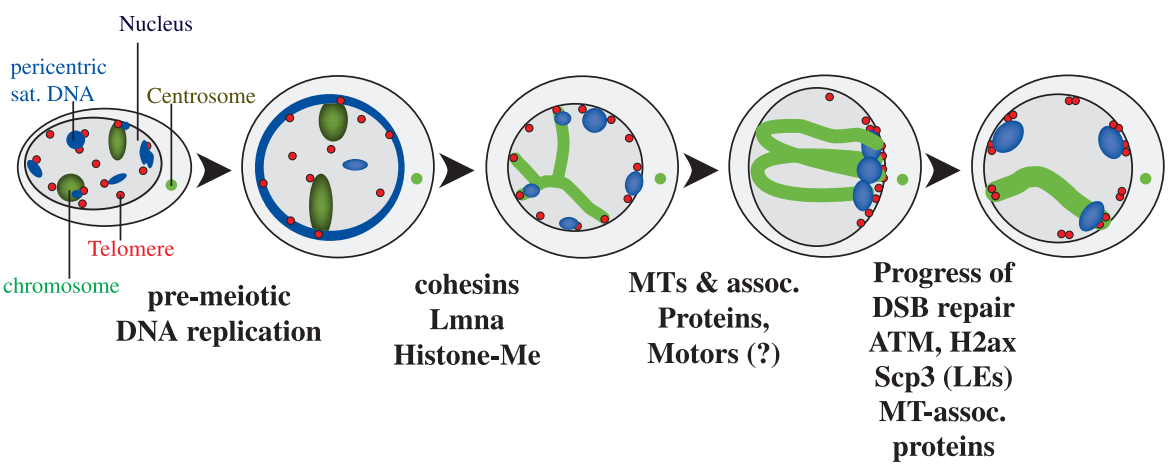

Fig. 2. A model of chromosome and telomere dynamics based on FISH staging of 3D-preserved early prophase I nuclei of the mouse (and other mammals; for details see Scherthan et al., 1996). Telomeres are shown in pink, pericentric major satellite DNA (pericentromeres) in blue and one pair (for simplicity) of acrocentric mouse chromosomes in green. Meiosis is a succession of stages and only the most striking nuclear topologies have been depicted. Premeiotic cells: Chromosomes are compact, spatially separated and satellite DNA forms numerous clusters that are distributed throughout the nuclear volume, as are the telomeres. Mid-preleptotene: After premeiotic DNA replication pericentromeric satellite DNA (pericentromeres) has largely relocated to the nuclear periphery (NP) which it is coating, while not all telomeres have transited to the NP yet. Chromosome territories start to elongate. Increase of mid-preleptotene spermatocytes in knockout mice deficient for A-type lamins and histone $\mathrm{H} 3-\mathrm{K} 9$ methylation suggest that these factors are required for timely transit through this stage. Leptotene: Telomeres of now elongated chromosomes have attached randomly over the NE, pericentric satellite DNA forms large peripheral clusters. Telomere repeats and proteins are likely required for peripheral telomere localization at this stage. Leptotene/zygotene transition: Telomeres congregate tightly opposite to the centrosome, which is a short-lived stage in the male mouse; evidence from mouse and other species suggests that this process likely requires non-cytoplasmic microtubules (MTs) and motor proteins. Directed telomere move- ments, oscillations and/or nuclear rotations could successively position telomeres near the centrosome, which will induce nuclear movements that instigate encounters among elongated chromosomes that carry double-stranded DNA breaks and phosphorylated histone H2AX. Leptotene and zygotene chromosomes undergo DSB-dependent homology testing and recognition. Telomeres get captured at the cluster site until progressed recombinational DSB repair and chromosome pairing elicit a release signal, possibly sensed by ATM and relayed through e.g. H2AX, to name one possibility. This leads to telomere dispersion during zygotene of mouse spermatogenesis, when synapsis, DNA repair and chromosome condensation progress further. Pachytene: All homologues are paired lengthwise (bivalents) and are connected by the synaptonemal complex. Telomeres are scattered over the nuclear envelope. Note: Absence or defective component(s) of meiotic chromosomes or DNA repair machinery may be lethal to meiocytes through response of a checkpoint that often manifests during pachytene or an equivalent stage. Increased frequencies or absence of a prophase I-specific nuclear motif indicates the requirement for a particular component (be it direct or indirect) in a prophase stage that precedes the stage affected. The components/properties given below the arrows indicate factors that contribute to the timely transit from one depicted stage to the next. Lack of a particular function will protract the preceding stage.

\title{
Erratum
}

Cytogenet Genome Res 108:365 (2005)

Erratum to Cytogenet Genome Res 106:43-48 (2004)

The most common chromosome aberration detected by high-resolution comparative genomic hybridization in vulvar intraepithelial neoplasia is not seen in vulvar squamous cell carcinoma

\author{
T. Bryndorf,, M. Kirchhoff, a J. Larsen, b B. Andreasson, , B. Bjerregaard, d \\ H. Westh, ${ }^{e}$ H. Rose ${ }^{a}$ and C. Lundsteen ${ }^{a}$ \\ ${ }^{a}$ Fertility Clinic, Rigshospitalet; ${ }^{b}$ Finsen Laboratory, Finsen Center, Rigshospitalet; \\ Departments of ${ }^{\mathrm{c}}$ Obstetrics and Gynecology and ${ }^{\mathrm{d}}$ Pathology, Herlev Hospital; \\ ${ }^{\mathrm{e}}$ Department of Clinical Microbiology, Hvidovre Hospital, University of Copenhagen (Denmark)
}

The affiliation for authors T. Bryndorf, M. Kirchhoff, H. Rose and C. Lundsteen was incorrect. 